\title{
Análisis de los discursos de médicos vertidos en Twitter sobre la validez de posibles tratamientos contra la COVID-19
}

\section{Discourse analysis of physicians on Twitter about the validity of possible treatments for COVID-19}

Alejandra Manco Vegaa

a Laboratorio ELICO, Departamento de Informática, Universidad Claude Bernard Lyon 1, Francia

\section{Resumen}

Introducción: Twitter es una plataforma que prioriza la inmediatez de la comunicación. Sin embargo, los tweets en áreas científicas casi nunca llegan a una audiencia amplia. Objetivo: Examinar estrategias discursivas utilizadas por médicos peruanos con una cuenta activa en Twitter sobre la validez de los posibles tratamientos contra la COVID-19. Metodología: Análisis crítico del discurso, mediante el uso del modelo tridimensional de Fairclough y con ayuda de las nociones de ethos. Resultados: Se encontraron dos ámbitos en los que se expresa el ethos discursivo médico-científico: la construcción como individuos especialistas en salud y el abordaje dado a los aspectos del ethos médico-científico (el método, la evidencia y lo pseudocientífico). Conclusiones: El lenguaje utilizado en los tweets es impersonal y neutro. El posicionamiento de los individuos como especialistas en salud se consigue mediante una opinión temática. Un ethos discursivo que refuerza el ethos prediscursivo existente a través de estrategias discursivas como la denominación y la intertextualidad es el que desarrolla este posicionamiento.

Palabras clave: Análisis crítico del discurso; Ethos; Twitter; Tratamientos contra la COVID-19; Médicos; Perú; Comunicación de la ciencia; Comunicación en salud.

\begin{abstract}
Introduction: Twitter is a platform that prioritizes the immediacy of communication. However, tweets in scientific areas rarely reach a wide audience. Objective: To examine discursive strategies used by Peruvian doctors with an active Twitter account on the validity of possible treatments for COVID-19. Methodology: Critical discourse analysis, using Fairclough's three-dimensional model with the help of notions of ethos. Results: Two areas were found where the medical-scientific discursive ethos is expressed: the construction as individual specialists in health and the approach given to the aspects of the medical-scientific ethos (the method, the evidence and the pseudo-scientific). Conclusions: The language used in the tweets is impersonal and neutral. The positioning of individuals as health specialists is done through a thematic opinion. A discursive ethos that reinforces the existing pre-discursive ethos through discursive strategies such as denomination and intertextuality is the one to develop this positioning.
\end{abstract}

Keywords: Critical discourse analysis; Ethos; Twitter; COVID-19 treatments; Physicians; Peru; Science communication; Health communication. 


\section{Introducción}

os discursos sobre ciencia y cómo esta puede contribuir a la sociedad son, hasta cierto punto, monopólicos, dado que solamente unas pocas personas tienen (o pueden tener) una opinión informada sobre este tema. Todas estas personas son científicos que trabajan en su respectivo campo, es decir, miembros de una pequeña élite dentro del país. En el contexto de la pandemia, se hace necesaria una información válida y precisa sobre el tema.

Este trabajo busca analizar estrategias discursivas utilizadas en Twitter por médicos e investigadores en salud para discutir sobre la validez de posibles tratamientos contra la COVID-19 a la fecha en la que se llevó a cabo esta investigación (el uso de la Cloroquina, Ivermectina, Remdesivir y Dexametasona). Esta plataforma social en línea desmediatiza la comunicación, de manera que los usuarios de esta red toman el control de sus canales oficiales de comunicación. El objetivo de este trabajo es analizar cómo los médicos e investigadores en salud enmarcan la importancia de la comunicación sobre los posibles tratamientos frente al coronavirus y cómo al hacer esto construyen su papel como especialista 0 científico en la sociedad. La hipótesis central de este trabajo es que las estrategias discursivas utilizadas por los especialistas se trasladan de la escritura académica a las plataformas sociales, dando forma a un ethos discursivo médico-científico en Twitter.

Sobre la escritura académica del área específica de medicina en idioma castellano, García Negroni (2008, p. 27) explica que existe una "total ausencia de mención directa de los discursos de los otros científicos, la constante alusión a las contribuciones e ideas de estos últimos bajo la forma de resultados alcanzados y el escaso porcentaje de comentarios meta-enunciativos sobre las diferentes no coincidencias del decir en este subcorpus". Esto explicaría el mayor efecto de "objetividad y neutralidad" asociado a las disciplinas llamadas "duras", y favorecería la representación del discurso de esta ciencia como medio "neutro y aséptico" de simple registro de los hechos. La autora (García Negroni, 2008) explica también que estas estrategias constituyen un ethos médico que busca describir objetos, experiencias y desarrollos de forma objetiva y transparente.

La comunicación de la ciencia surge como una forma de institucionalización del sector de la investigación y el crecimiento de los medios de comunicación. Asimismo, existe un discurso consensuado sobre la idea de que la ciencia y la actividad científica son beneficiosas para la sociedad (Bucchi, 2008). La concepción tradicional implica una visión paternalista de la comunicación científica. Bucchi (2008) argumenta que esta visión tradicional de la comunicación científica implica la incapacidad del público para comprender y apreciar los logros de la ciencia, debido a una hostilidad pública perjudicial. Esta teoría difusionista ve a las personas como meros receptores de información, cuya ignorancia y hostilidad por defecto hacia la ciencia pueden contrarrestarse mediante la inyección apropiada de comunicación científica. En este caso, el proceso de comunicación científica comienza con el especialista y termina en el discurso popular. Como este concepto tradicional de comunicación pública de la ciencia es el más aceptado y conocido, Bucchi $(2008$, p. 57$)$ argumenta que también es una ideología presente en este tema.

La plataforma Twitter es una de las redes sociales más populares, con millones de usuarios alrededor del mundo (López-Goñi \& Sánchez-Angulo, 2018). En un contexto de "noticias falsas" y "post-verdades", la comunicación de la ciencia por medios accesibles como Twitter u otras redes sociales se vuelve transcendental (Patel, 2019). Al respecto, Pérez-Rodríguez, González-Pedraz, \& Berrocal (2018) encuentran que, para el caso específico de la comunicación de ciencia en Twitter, son los propios científicos quienes por medio de sus perfiles personales realizan este trabajo de difusión, y también reciben mayor visibilidad gracias a los retuits en esta red.

Las principales ventajas que ofrece Twitter para los científicos, según López-Goñi \& Sánchez-Angulo (2018) son: incrementar su branding personal, mejorar sus habilidades de comunicación escrita, y promover el involucramiento del público con la ciencia. Una conclusión a que la también llegan Budge, Lemon \& McPherson (2016), quienes argumentan que los académicos usan Twitter como parte de su proyección como especialista o autoridad de manera pública, así como una forma de hacer accesible la producción académica.

Sobre los resultados de la comunicación de la ciencia en Twitter, Bombaci, Farr, Gallo, Mangan, Stinson, Kaushik \& Pejchar (2015) sostienen que los tweets casi nunca llegan a una audiencia de formuladores de políticas, organismos gubernamentales u ONGs, a pesar de la intención de los presentadores. En la mayoría de los casos, la audiencia de los tweets casi siempre está constituida por otros pares (miembros de la academia, investigadores, otros científicos), y solo ocasionalmente por comunicadores.

Twitter es también la red social predominante para compartir información concerniente a temas de la salud, pero también en donde se distribuye desinformación (Lopez-Pujalte \& Nuño-Moral, 2020). De este modo, por ejemplo, el artículo de Gonsalves, McGannon, \& 
Pegoraro (2019) discute cómo la Fundación Canadiense para el Corazón y los Accidentes Cerebrovasculares y los usuarios canadienses de Twitter vinculan las experiencias e identidades de las mujeres con la campaña de concienciación sobre enfermedades cardiovasculares \#MoreMoments, utilizando un enfoque de análisis crítico del discurso. Estas autoras (Gonsalves et al., 2019) identifican microelementos de un texto a los que denominan "pequeñas historias", dentro de extractos de un hashtag de Twitter. En este trabajo, las autoras $(2019$, p. 3 ) identifican las posiciones de los sujetos y determinan la construcción de los agentes sociales en las dinámicas de poder y los contextos políticos, así como los derechos de expresión y los roles de comportamiento y cómo las responsabilidades relacionadas con la salud apoyaron o resistieron estas construcciones (Gonsalves et al., 2019, p. 16).

De igual forma, Rochwerg et al. (2020) mencionan que la vasta información en salud disponible en Twitter y la rapidez de la actualización de esta plataforma ocasionan que dentro de esta plataforma coexistan información proveniente de fuentes fiables y conjeturas no comprobadas. Además, Twitter puede también provocar histeria e influir de manera negativa en el bienestar mental de sus usuarios (Rosenberg, Syed, \& Rezaie, 2020).

Twitter es una herramienta de información y comunicación fundamental en el contexto de la crisis por la COVID-19. Wicke \& Bolognesi (2020) estudian el discurso público en Twitter sobre la COVID-19 durante marzo y abril del 2020. Estos autores identifican temas recurrentes en el corpus de tweets identificados para este estudio, como por ejemplo: comunidad y compasión comunitaria; política y reacción a la epidemia; y, el más importante de todos, el marco figurativo de guerra. En la misma línea, Pérez-Dasilva, Meso-Ayerdi \& MendigurenGaldospín (2020) concluyen que, ya para finales de febrero de 2020, la COVID 19 pasa a generar una crisis de información por la aparición de bulos sobre este tema en esta plataforma en línea

También existen trabajos sobre el uso de Twitter por parte de médicos. Morandeira Rivas, Riquelme Gaona, Álvarez Gallego, Targarona Soler \& Moreno Sanz (2018) realizaron una encuesta sobre el uso de las redes sociales por parte de los cirujanos generales en España. Estos autores encontraron que los cirujanos de menor edad tienen más posibilidad de tener un perfil en redes sociales. También encontraron que el uso profesional de las redes sociales se centra en la difusión de información sobre eventos científicos, actividades educativas, actualización de conocimientos y comunicación entre expertos (Morandeira Rivas et al., 2018).
Durante el año 2020 se han publicado varios artículos que estudian el uso de Twitter por parte de los médicos e investigadores en salud para abordar temas relacionados con la COVID-19. De acuerdo a Pollet \& Rivers (2020) y Rochwerg et al. (2020), Twitter se ha consolidado como un lugar de encuentro y una fuente de información para el intercambio científico durante las emergencias de salud pública por su rapidez en la diseminación de datos sobre la COVID-19 entre los investigadores. Del mismo modo, para Ghosh, Schwartz \& Narouze (2020), Twitter facilita la colaboración instantánea y remota con otros médicos en el contexto de la pandemia. Por otra parte, el trabajo de Wahbeh, Nasralah, Al-Ramahi \& El-Gayar (2020) detalla que los médicos tuitean sobre ocho categorías principales relacionadas con la COVID-19: acciones y recomendaciones, lucha contra la desinformación, sistema de salud, información y conocimiento, síntomas y enfermedad, inmunidad, testeo, infección y transmisión. Las categorías de acciones y recomendaciones y la lucha contra la desinformación suponen casi el $50 \%$ del total del corpus de los tweets revisados para esta investigación.

\section{Métodos}

Para este análisis del discurso se utilizó el modelo tridimensional de Fairclough (2006), que consta de: el texto, en el que se analizan datos transcritos; la práctica discursiva, en la que se pueden analizar los procesos de producción, relaciones y consumo; y, finalmente, la práctica social, donde se analizan las estructuras sociales subyacentes. También se contó con la ayuda de las nociones del ethos de Amossy (2016) y Maingueneau (2002). El ethos es un concepto de difícil definición, ya que es una construcción lingüística pero también una posición institucional (Amossy, 2016). Asimismo, un ethos es compartido por miembros de una comunidad (Maingueneau, 2002). Para este trabajo, se utiliza la distinción de Maingueneau (2002) entre ethos discursivo y pre-discursivo, de manera que el ethos prediscursivo es una imagen prediseñada del orador.

Ambos marcos metodológicos son complementarios, pues como argumenta Ramírez (2013), a pesar de que ambos esquemas analíticos destaquen la importancia del poder como elemento central del análisis, el proceso del mismo privilegia distintos aspectos. La elección de ambos marcos teóricos se debe a que los médicos e investigadores en salud no ocupan un lugar dominante dentro de la sociedad peruana, pero sí cuentan con una posición de autoridad en su campo científico por el mismo hecho de su trabajo. 
La elección del material fue motivada considerando su relevancia para la pregunta de investigación y su acceso a través de Internet. El material de este trabajo está compuesto por lenguaje escrito, disponible en tweets individuales sobre el tema. Se trata de discursos escritos en Twitter. En especial, se privilegió al análisis de "pequeñas historias" (Georgakopoulou, 2015), que son interacciones puntuales sobre un tema y en un contexto específicos, dado que Twitter facilita una forma miniaturizada de comunicación (Georgakopoulou, 2015).

El rango de fechas corresponde al inicio y fin de la cuarentena por la pandemia de la COVID-19 en Perú, desde el 15 de marzo al 30 de junio de 2020. La selección de las cuentas fue hecha con ayuda del algoritmo de Twitter "A quién seguir", que sugiere perfiles similares. Para esta selección inicial de cuentas personales de Twitter se utilizaron cuatro criterios: 1) cuentas activas con tweets propios que generen cierta interacción; 2) cuentas que hablen sobre la pandemia en Perú y sus posibles tratamientos en idioma castellano; 3) la autopresentación de cada cuenta individual corresponde a un médico o investigador en salud peruano radicado en Perú o en el extranjero; 4) cuentas que en el momento de la selección no trabajaban en un cargo público y cuentan con publicaciones académicas sobre el tema de la salud.

Las cuentas analizadas, su autopresentación y número de seguidores se detallan en la Tabla 1.

Las cadenas de búsqueda dentro de estas cuentas fueron: hidroxicloroquina (from:Cuenta), hidrocloroquina (from:Cuenta), cloroquina (from:Cuenta), ivermectina (from:Cuenta), remdisivir (from:Cuenta), dexametasona (from:Cuenta). Finalmente, los 33 tweets analizados de cada una de las cuentas seleccionadas se encuentran detallados en el anexo $A$.

Tabla 1. Características de las cuentas de Twitter seleccionadas.

\begin{tabular}{|c|c|c|c|}
\hline Cuenta & Autopresentación & $\begin{array}{l}\text { Número de } \\
\text { seguidores }\end{array}$ & $\begin{array}{c}\text { Número } \\
\text { de tweets } \\
\text { seleccionados }\end{array}$ \\
\hline @Alvaro_Taype & $\begin{array}{l}\text { Médico epidemiólogo / Memes / Ser el eterno } \\
\text { forastero, el eterno aprendiz, el eterno postulante }\end{array}$ & 1.066 & 16 \\
\hline @drhuerta & $\begin{array}{l}\text { Médico y comunicador. Los lugares más oscuros } \\
\text { del infierno están reservados para aquellos que } \\
\text { mantienen su neutralidad en tiempos de crisis } \\
\text { moral. Dante. }\end{array}$ & $138 \mathrm{mil}$ & 7 \\
\hline @emezones & $\begin{array}{l}\text { Physician, Scientist and Epidemiologist. } \\
\text { Independent Consultant, Professor and } \\
\text { Researcher. Peruvian Citizen }\end{array}$ & 772 & 4 \\
\hline $\begin{array}{l}@ \\
\text { ErnesBustamante }\end{array}$ & $\begin{array}{l}\text { Molecular Biologist \& Biochemist. PhD by Johns } \\
\text { Hopkins Univ. Medical School; BS \& MS Cayetano } \\
\text { Heredia Univ. DNA Personalized Medicine. Ex- } \\
\text { Chief Peru's NIH (INS) }\end{array}$ & $15,8 \mathrm{mil}$ & 4 \\
\hline @jjaimemiranda & $\begin{array}{l}\text { MD PhD Epidemiology NCDs Global Health } \\
\text { @cronicasperu } \\
\text { @MEDICINA_UPCH } \\
\text { @CayetanoHeredia } \\
\text { Globo terráqueo con Europa y África Visiting Prof } \\
\text { @georgeinstitute } \\
\text {,Prof } \\
\text { @LSHTM } \\
\text {, Lown Scholar } \\
\text { @HarvardChanSP }\end{array}$ & 4.993 & 2 \\
\hline
\end{tabular}




\section{Resultados}

Los textos revisados fueron escritos en el contexto de la pandemia en Perú. En este caso, para la comunicación de un tema tan especializado, como es la validez de un posible tratamiento médico, se adopta una teoría difusionista de la comunicación de la ciencia (Bucchi, 2008). Esto se refuerza con el hecho de que muchos de los tweets revisados no generan mucha interacción o conversaciones con otros usuarios más allá que con otros pares. Este mismo fenómeno sucede tanto en las cuentas con pocos seguidores como en las dos cuentas consolidadas con miles de seguidores, como lo son @ drhuerta y @ErnesBustamante.

En las pocas ocasiones donde sí hay respuestas por parte de los autores, la interacción está escrita en modo lejano y formal. Por ejemplo, @ErnesBustamante trata de usted a los comentaristas, o es el mismo autor quien hace un hilo tras el tweet inicial. Otra característica que se resalta es el poco uso, casi nulo, de hashtags por estas cuentas.

El ethos prediscursivo precede a la construcción de la imagen en el discurso (Amossy, 2016). Una persona legitimada, es una autoridad exterior de la que goza el locutor, que se percibe como "un portavoz autorizado" (Amossy, 2016). Por lo tanto, el locutor tiene un capital simbólico acumulado, ya que el que habla tiene poder a los ojos del público (Amossy, 2016). Por lo mismo, el ethos prediscursivo y el capital simbólico que conlleva se posicionan como una práctica social. En los casos revisados, el ethos prediscursivo (la imagen prediseñada del otro) tienen límites borrosos con el ethos discursivo. El ethos prediscursivo de los médicos e investigadores en salud se confirma por medio del ethos discursivo en Twitter.

El ethos prediscursivo contiene un estereotipo, que consiste en pensar en lo real por medio de una representación cultural preexistente (Amossy, 2016). El ethos prediscursivo de los médicos y los científicos es el de alguien serio y estudioso que busca encontrar respuestas correctas y basadas en la evidencia para preguntas "científicas". Otro de los estereotipos de los científicos es que trabajan en una "torre de marfil", es decir, un ambiente altamente especializado. En este sentido, Gimenes \& Mira (2019) proponen que el ethos prediscursivo médico consiste en una posición de autoridad en el tema como una persona calificada que puede responder a todas las preguntas y ser confiable en términos de capacitación, apoyo profesional y con una actitud digna de confianza.
Normalmente, el discurso sobre lo científico tiene un espectro lejano y serio, y en estas circunstancias especiales también. Esto se debe sobre todo a la selección léxica utilizada por los autores, y también la importancia suprema dada al método científico como diferenciador de lo que los autores hacen, cumplen y saben (y lo que los demás usuarios de esta plataforma no). Dado que el orador, en este caso el escritor en Twitter, construye su propia imagen en función de la imagen que el auditorio tiene de él/ella (Amossy, 2016), esta autopresentación refuerza el estereotipo anterior.

Se encontraron dos ámbitos en los que se expresa la pretensión de neutralidad propia de escritura académica de este campo científico (García Negroni, 2008), que son: la construcción como individuos especialistas en salud y el abordaje dado a los aspectos del ethos médicocientífico (el método, la evidencia y lo pseudocientífico).

Posicionamiento de los individuos como especialistas en salud

El posicionamiento como figuras de autoridad en el tema en Twitter se consigue a través de una opinión sobre un tema específico. Una toma de postura reiterada y a lo largo de un tiempo específico crea identidades (Evans, 2016). En los casos revisados, la mayoría de usuarios se encuentra en contra del tratamiento propuesto por el Ministerio de Salud (MINSA). Se trata de una opinión crítica que da a entender que el hablante (el especialista) podría hacerlo mejor, lo que demuestra la existencia de un celo profesional.

Se utiliza mucho la denominación del sistema de representación de actores sociales para hacer referencia a colegas investigadores, portales o instituciones de investigación y organismos públicos, con el fin de resaltar la autoridad, credibilidad y agencia de los que son nombrados, como por ejemplo: el MINSA, el Colegio Americano de Médicos, la Administración de Medicamentos y Alimentos (FDA, por sus siglas en inglés), la Red de Farmacovigilancia de Francia, etc. Es decir, los otros actores sociales de la comunidad del ethos prediscursivo se explicitan en el discurso.

De la misma manera, la referencia a organismos de investigación y universidades de países occidentales (EE.UU., Francia, etc.) es constante. Esta denominación se utiliza como un recurso para comunicar que un estudio desarrollado 0 una medida adoptada allí tienen valor. A continuación, se puede observar un par de ejemplos sobre la denominación de la FDA en la Figura 1 y la Figura 2. 


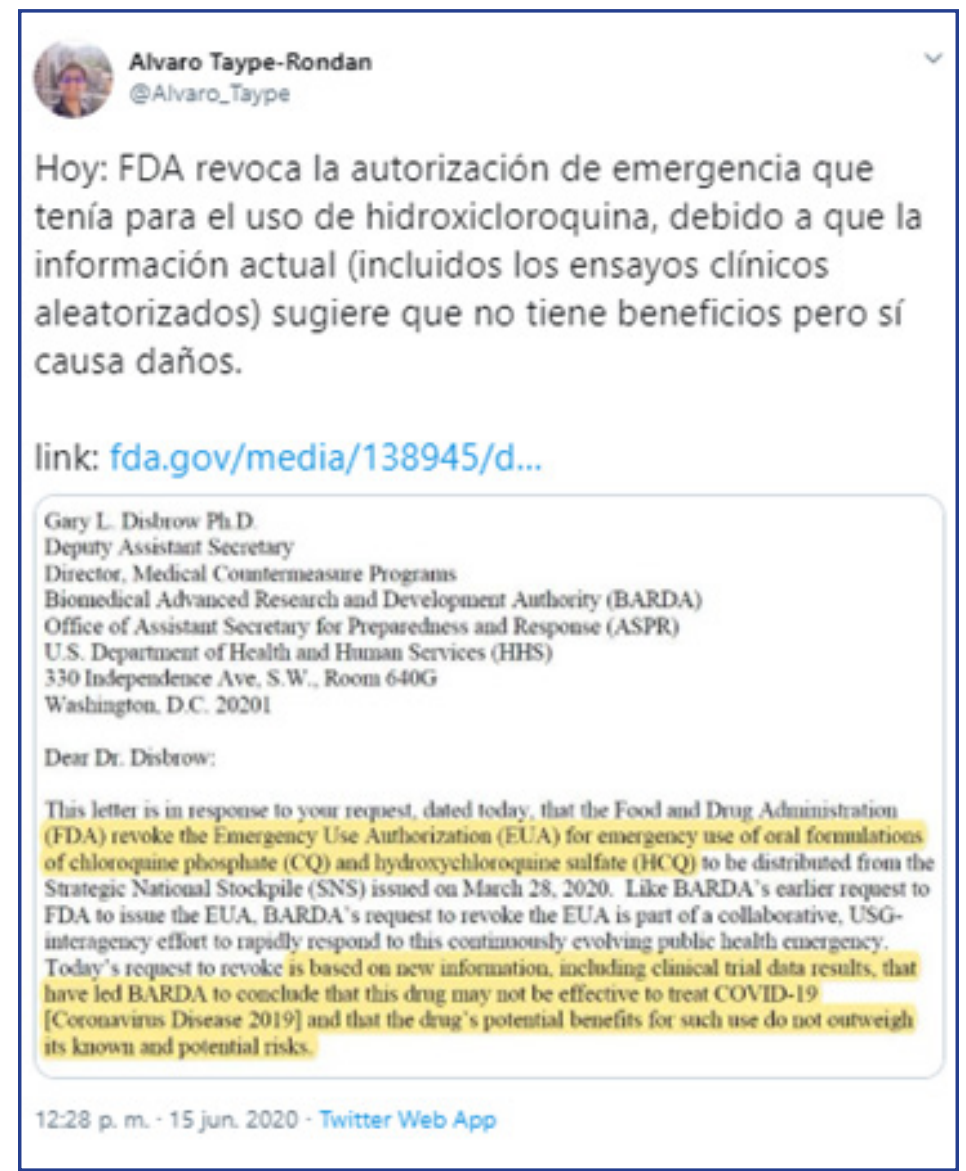

Figura 1. Alvaro Taype-Rondan "Hoy: FDA revoca la autorización de emergencia que tenía para el uso de hidroxicloroquina, debido a que la información actual (incluidos los ensayos clínicos aleatorizados) sugiere que no tiene beneficios pero sí causa daños". https://twitter.com/Alvaro_Taype/status/1272581844404514819

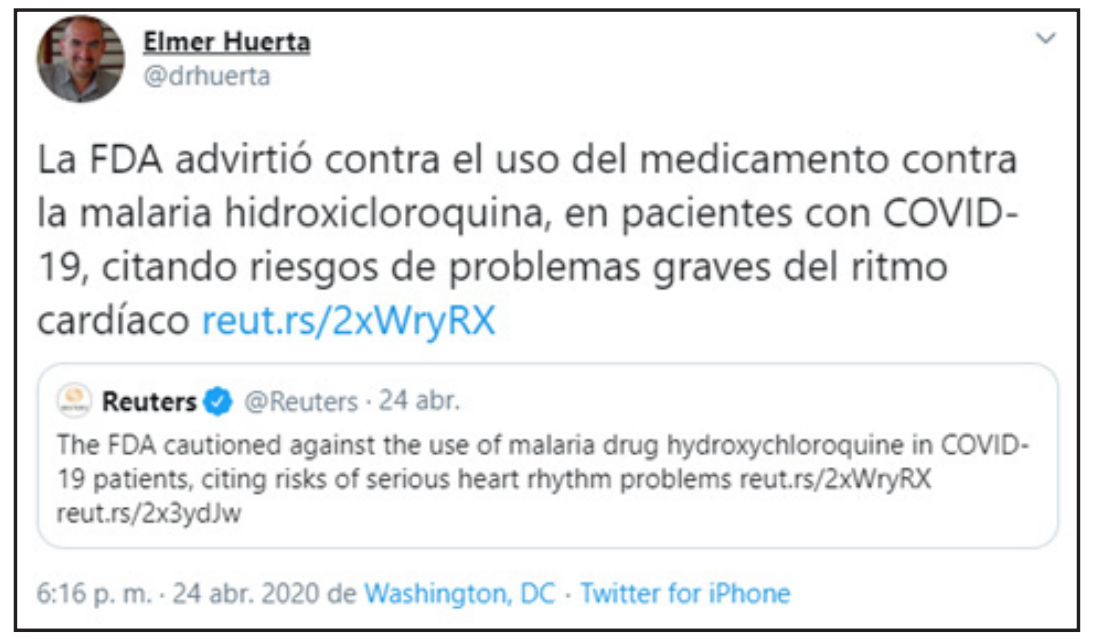

Figura 2. Elmer Huerta: "La FDA advirtió contra el uso del medicamento contra la malaria hidroxicloroquina, en pacientes con COVID-19, citando riesgos de problemas graves del ritmo cardíaco". https://twitter.com/drhuerta/ status/1253825155324956673 
Asimismo, este énfasis y hasta sobreuso de la denominación es una estrategia que brinda legitimación y credibilidad a las entidades (personas e instituciones) nombradas, al mismo tiempo que construye un ethos médico científico discursivo. Este ethos (Bermúdez, 2007) se configura entonces como una construcción de identidad de estos sujetos usuarios de Twitter como figuras especialistas, a partir de la referencia a la credibilidad del colectivo de estas otras entidades denominadas.

A lo largo de la mayoría de los tweets revisados, también resalta el uso de la intertextualidad que se manifiesta con el uso de verbos reportativos por medio de una atribución directa (Figura 1 o Figura 2) o el uso de comillas (Figura 3). En estos casos, esta estrategia discursiva se utiliza para resaltar algo que otra persona o entidad importante menciona, lo que ocasiona una idealización de la imagen de estas personas o entidades. Aquí se utiliza el efecto de objetivación y de autoridad, citando a gente y entidades importantes en el campo científico. También se utiliza la atribución directa, que otorga identificación y visibilidad a la persona o entidad a la que se cita, lo que a su vez refuerza la pretensión de neutralidad del ethos prediscursivo y la credibilidad del ethos discursivo.

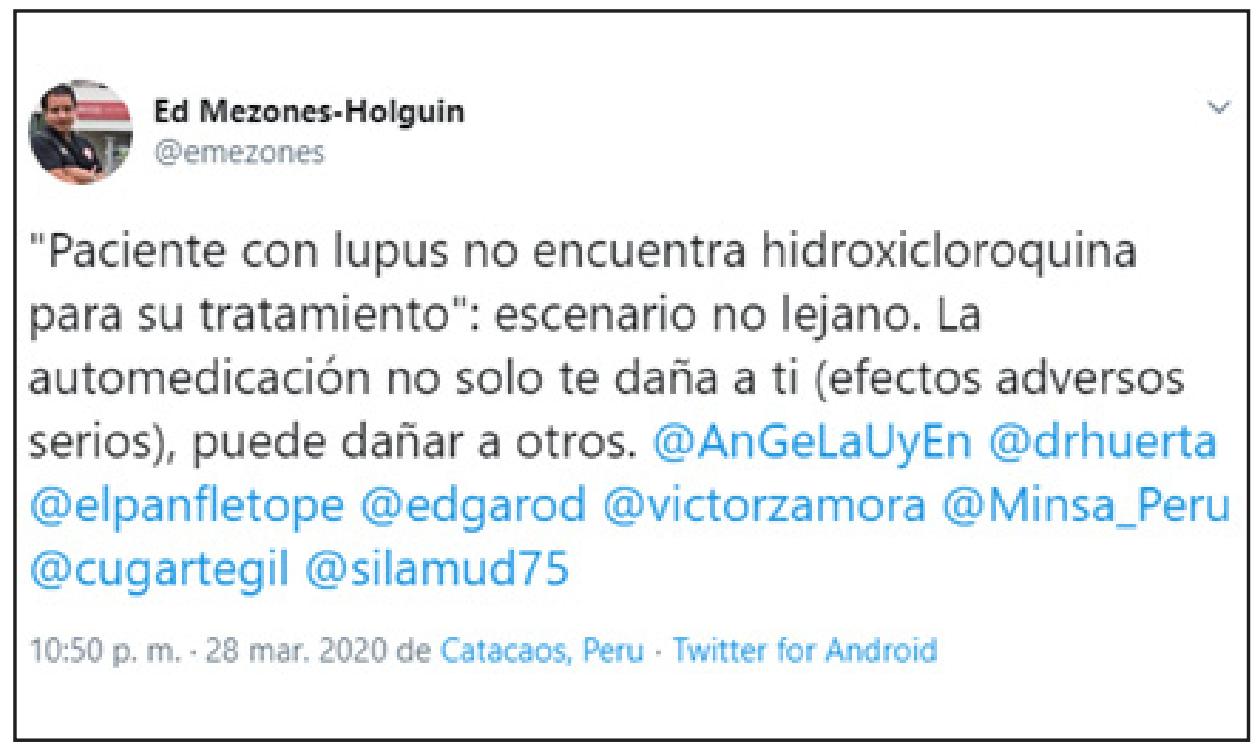

Figura 3. Ed Mezones-Holguín: "Paciente con lupus no encuentra hidroxicloroquina para su tratamiento". https:// twitter.com/emezones/status/1244109742236209159 
Aspectos del ethos médico-científico: el método, la evidencia, lo pseudocientífico

La autopresentación de sí mismo al público en Twitter en los casos revisados es como un especialista en salud. Para hacerlo, se recurre a las explicaciones detalladas del ámbito profesional, como son el método científico, la evidencia y lo que constituye lo pseudocientífico. De esta manera, se señalan el ámbito de lo científico y todos los conceptos que este incluye (la metodología, la publicación de resultados con un proceso de revisión por pares, la evidencia) y, por último, lo que no es científico (lo pseudocientífico).

En la Figura 4 se comparte un artículo sobre el excepcionalismo en la investigación, en el que el autor utiliza la exclusión-supresión dentro de una metáfora: [Los científicos] reclaman ciencia dura para conseguir estudios robustos y no anécdotas. La selección léxica es interesante pues se habla de criterios específicos, ciertamente científicos, que deben ser cumplidos, ya que de lo contrario lo que se obtiene no es nada válido. Por otro lado, el "nosotros" del ethos [nosotros, los científicos] marca la importancia de seguir estos criterios para este grupo de personas.

Otro aspecto importante que se ha resaltado es el proceso de revisión por pares, a través de un lenguaje normativo, así como el principio de reproducibilidad. También se resalta la importancia de la evidencia en el quehacer científico. Por ejemplo, para la construcción del concepto de "medicina basada en la evidencia" se utiliza una metonimia cambiando la palabra por otra: vehemencia (Figura 5). Igualmente, en la Figura 6 también se resalta la palabra "evidencia" (o mejor dicho su falta).

Otra palabra que se repite es la de "pseudociencia". Se denomina pseudociencia a todo lo que no ha pasado por los criterios del método científico mencionados anteriormente, como por ejemplo, el uso de suplementos de vitamina C (Figura 7). El concepto de pseudociencia se construye en referencia a lo que no es científico y lo que va en contra del ethos científico anteriormente mencionado.

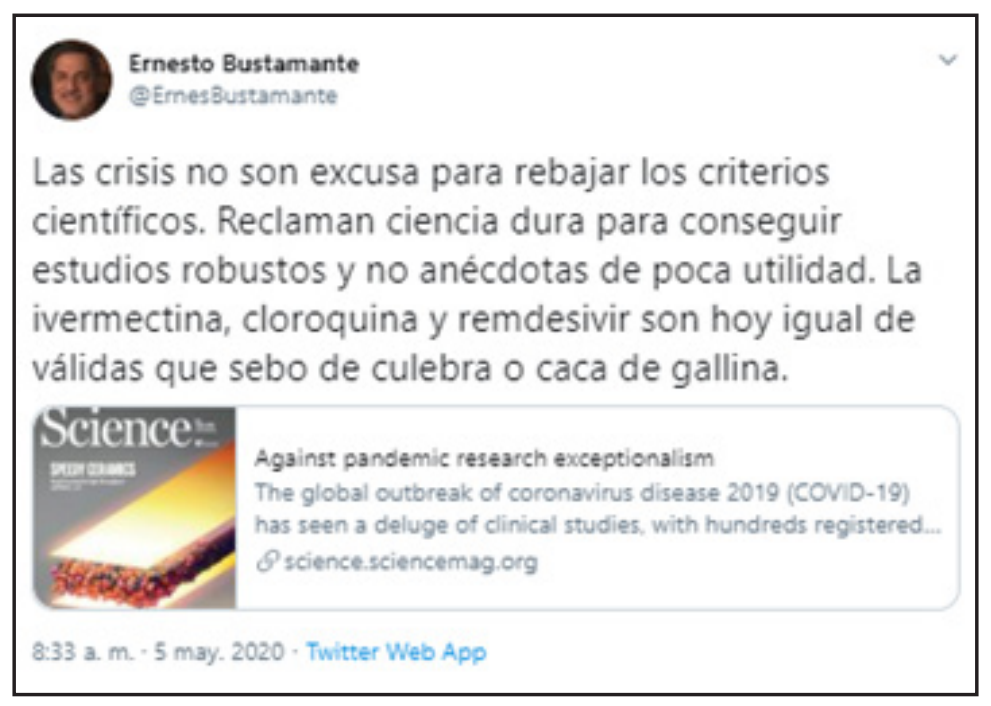

Figura 4. Ernesto Bustamante: "Las crisis no son excusa para rebajar los criterios científicos". https://twitter.com/ ErnesBustamante/status/1257664768887992321 
Rev Esp Comun Salud // 83

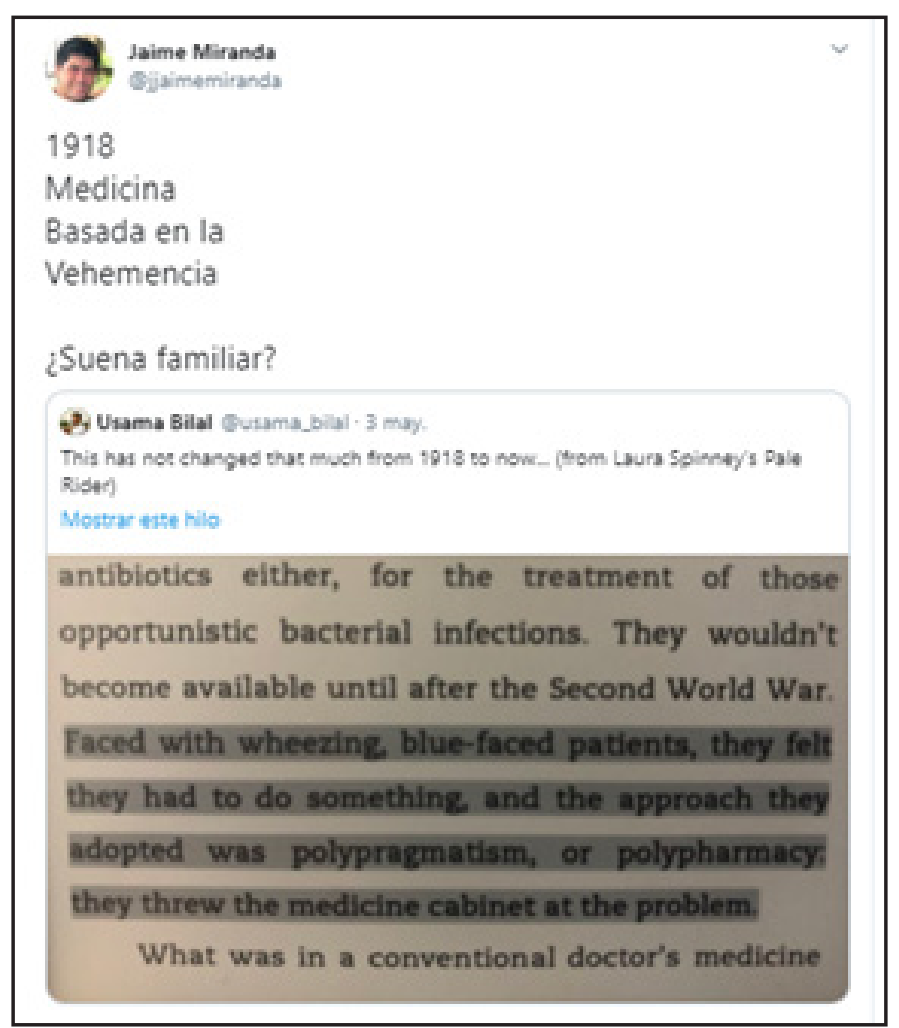

Figura 5. Jaime Miranda: "1918 Medicina Basada en la Vehemencia ¿Suena familiar?”. https://twitter.com/ jjaimemiranda/status/1257160765896749057

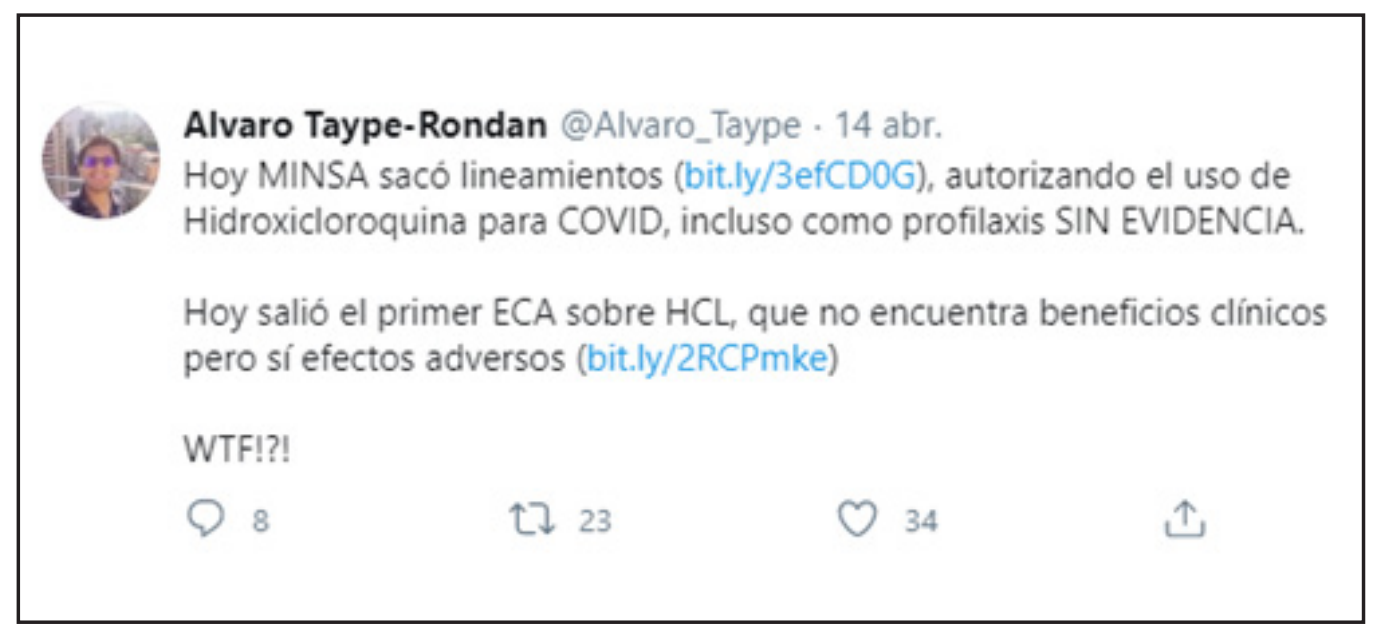

Figura 6. Alvaro Taype-Rondan: "Hoy MINSA sacó lineamientos, autorizando el uso de Hidroxicloroquina para COVID, incluso como profilaxis SIN EVIDENCIA". https://twitter.com/Alvaro_Taype/status/1250203161899474951 


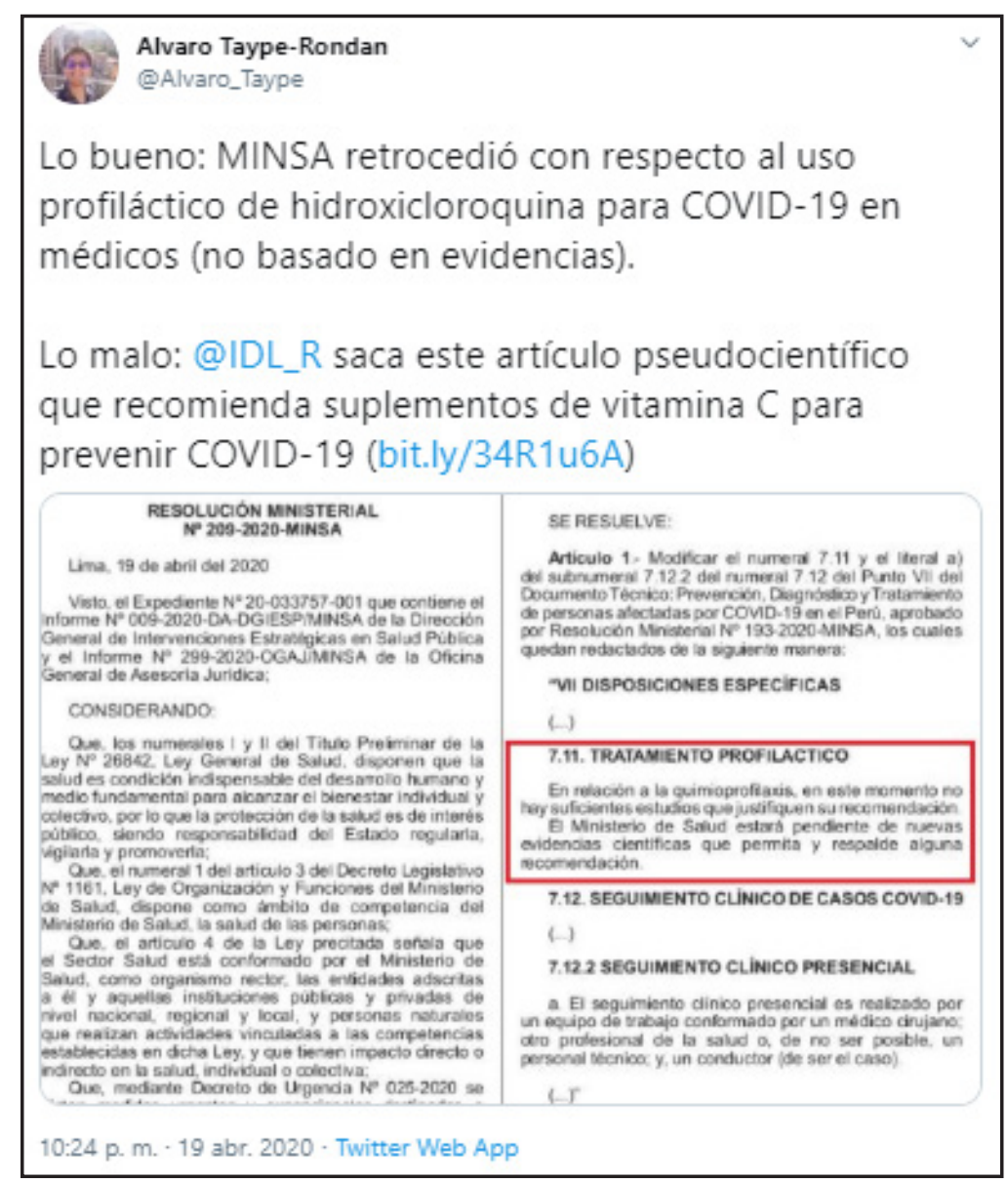

Figura 7. Alvaro Taype-Rondan: "Lo bueno: MINSA retrocedió con respecto al uso profiláctico de hidroxicloroquina para COVID-19 en médicos (no basado en evidencias). Lo malo: @IDL_R saca este artículo pseudocientífico que recomienda suplementos de vitamina C para prevenir COVID-19". https://twitter.com/Alvaro_Taype/status/1252075742806126593

\section{Práctica social}

La práctica social resultante de estas estrategias discursivas se da en el contexto de una sociedad neoliberal como la peruana, donde históricamente el comercio, el campo económico y las personas que trabajan en este campo específico ocupan el lugar más importante dentro de la sociedad. Por lo tanto, los científicos no tienen una posición de poder entre la élite de la sociedad. Esto se debe a que el país no es industrializado, es decir, el enfoque económico tiende a centrarse en la extracción y exportación de materias primas y por lo tanto las personas que trabajan en el campo de la Investigación y Desarrollo $(I+D)$ no ocupan un posicionamiento importante.

De esta manera, la posición y el capital simbólico que tienen los científicos son bastante modestos. Esta situación se produce, y a la vez se refuerza, por las estrategias discursivas utilizadas que dificultan la comunicación con un gran público. Esto termina contribuyendo a la reproducción de las inequidades ya existentes en términos de consumo y producción de información científica y en líneas más generales, en la educación terciaria.

\section{Discusión}

A pesar de las múltiples ventajas que ofrece Twitter como medio de comunicación de la ciencia para los científicos (López-Goñi \& Sánchez-Angulo, 2018; Budge, Lemon \& McPherson, 2016), especialmente para la comunicación pública de la ciencia para el gran público, este trabajo encuentra que los tweets revisados no generan mucha interacción o conversaciones con otros usuarios más allá que con otros pares, lo cual concuerda con los resultados del trabajo de Bombaci et al. (2015).

El trabajo de Morandeira Rivas et al. (2018) encuentra que los cirujanos jóvenes tienen mayores posibilidades de tener perfiles de redes sociales activos. Sin embargo, este trabajo encuentra que los perfiles de médicos e investigadores en salud con una carrera consolidada que 
participan en otros medios de comunicación (como la televisión abierta, de cable o la radio) y al mismo tiempo escriben en su cuenta de Twitter, cuentan con muchos más seguidores que sus pares más jóvenes y sin tanta exposición mediática.

Si bien los mensajes se han escrito en la plataforma Twitter durante esta circunstancia específica, la pandemia, se puede afirmar que el lenguaje utilizado es impersonal y neutro. Esto parece ser una traslación de la pretensión de neutralidad del estilo propio de la escritura científica en artículos académicos de esta área específica (García Negroni, 2008) hacia los tweets. Esto es una manifestación de un ethos preexistente que a la vez dificulta la comunicación de la ciencia, la cual termina adoptando un enfoque difusionista.

Lo más resaltable que se encontró es que, a diferencia de la escritura académica en medicina, donde casi no se menciona directamente los discursos de colegas (García Negroni, 2008), en Twitter se hace uso de la denominación constantemente. Se menciona directamente a otros organismos del área $u$ otros científicos, así como a usuarios de Twitter. También se utiliza la intertextualidad, mediante una atribución directa empleando verbos reportativos, pero siempre relacionados a este campo de estudio específico.

El tema de la desinformación es omnipresente a lo largo de la colección de tweets revisados, pero no se le denomina directamente así, sino que para nombrarlo se introduce el concepto de lo pseudocientífico, lo que no cumple con los criterios del método científico. Esta definición coincide con la que encontraron Salvador Mata, Raffio \& Cortiñas-Rovira (2020, p.73) en su trabajo sobre la percepción de las pseudociencias en el colectivo médico español: "la falta de rigor científico en la demostración de sus afirmaciones, que no derivan de ensayos clínicos representativos y realizados por métodos científicamente avalados". Este hecho también coincide con las conclusiones del trabajo de Wahbeh et al. (2020), quienes arguyen que este tema es una gran preocupación para los profesionales de la medicina activos en Twitter.

Otro punto importante que resaltar es que a pesar de que el trabajo de Gimenes \& Mira (2019) analiza interacciones producidas personalmente (cara a cara), y encuentra que el ethos prediscursivo se funde con el discursivo en el caso de los médicos, los resultados son parecidos a los de este trabajo que analiza interacciones puntuales en Twitter. Esto hace suponer que el ethos prediscursivo se puede manifestar en diversos formatos (interacción presencial o plataforma social en línea) casi sin cambio alguno y que también, el entorno de plataformas de redes sociales y lo oral tienen características comunes en cuanto a la facilidad de comunicación y las estrategias utilizadas para ello.

\section{Conclusiones}

Este trabajo encontró que las estrategias discursivas utilizadas por médicos en Twitter difieren notablemente de la escritura académica en esta especialidad. Es decir, el ethos discursivo médico-científico es alimentado por otro tipo de estrategias en esta red social en línea, especialmente la denominación e intertextualidad, que de igual manera refuerzan la pretensión de neutralidad del ethos prediscursivo y resaltan la credibilidad del ethos discursivo.

Existen dos ámbitos en los que el ethos discursivo se expresa: la construcción como individuos especialistas en salud y el abordaje dado a los aspectos del ethos médicocientífico (el método, la evidencia y lo pseudocientífico). El posicionamiento de los individuos como especialistas en salud se consigue mediante una opinión temática. Este posicionamiento se desarrolla mediante un ethos discursivo que refuerza el ethos prediscursivo existente mediante la denominación y la intertextualidad. Otros aspectos del ethos médico-científico, como son la metodología, la evidencia y lo pseudocientífico, son desarrollados por medio de la selección léxica y contribuyen al desarrollo de la autopresentación del ethos discursivo.

Este hecho genera que, a pesar de las ventajas que una plataforma participativa como Twitter ofrece, estas seas infrautilizadas a efectos de comunicación de la ciencia. Esto contribuye a la reproducción de las inequidades existentes en términos de consumo de información científica, pues los escritores recurren al concepto tradicional de comunicación de la ciencia, la teoría difusionista. Se encontró también que los tweets revisados, que contienen información sobre los posibles tratamientos del COVID-19, no generan mucha interacción en la plataforma de Twitter más allá que con otros pares. El lenguaje formal y lejano es utilizado en estas interacciones puntuales. En general, el lenguaje utilizado en los tweets revisados es impersonal y neutro, lo cual parece ser una traslación de la pretensión de neutralidad del estilo propio de la escritura científica en artículos académicos, pero que en realidad se trata de la presencia del ethos prediscursivo.

Finalmente, este estudio cuenta con dos limitaciones claras: 1) se utiliza un microanálisis textual de corte cualitativo, lo cual tiene limitaciones en cuanto al tamaño 
de la muestra y su posible generalización, 2) la selección de cuentas para analizar se hizo con ayuda del algoritmo de Twitter "A quién seguir", que no es neutro sino que depende de las variables incluidas en este algoritmo, lo cual puede haber introducido un sesgo subyacente en este trabajo. Por lo tanto, próximas investigaciones podrían revisar el tema con otro tipo de muestreo para comprobar (o no) si las mismas estrategias discursivas para la comunicación de la ciencia se utilizan en Twitter 0 en otros contextos. También se puede realizar una aproximación diferente, como por ejemplo una metodología cuantitativa como la minería de datos para el análisis textual, como es el caso del trabajo de Wicke \& Bolognesi (2020).

\section{Financiación}

Esta investigación no recibió financiación alguna.

Declaración de disponibilidad de datos

Los datos presentados en este estudio pueden ser solicitados a la autora de correspondencia.

\section{Agradecimientos}

Se agradece a todas las personas que revisaron este trabajo y brindaron sus perspectivas en las distintas etapas de preparación del manuscrito.

\section{Conflicto de interés}

La autora declara que no existe un conflicto de interés.

\section{Referencias bibliográficas}

Amossy, R. (2016). O ethos na intersecção das disciplinas: retórica, pragmática, sociologia dos campos. En: R. Amossy, (Ed.) Imagens de si no discurso: a construção do ethos (pp. 119-144). São Paulo: Contexto.

Bermúdez, N. (2007). Noción de ethos: historia y operatividad analítica. Tonos: Revista Electrónica de Estudios Filológicos, 14. Recuperado de: https:// www.um.es/tonosdigital/znum14/secciones/estudios1-ethos.htm

Bombaci, S. P., Farr, C. M., Gallo, H. T., Mangan, A. M., Stinson, L. T., Kaushik, M., \& Pejchar, L. (2015). Using Twitter to communicate conservation science from a professional conference. Conservation Biology, 30(1), 216-225. doi:10.1111/cobi.12570

Budge, K., Lemon, N. \& McPherson, M. (2016). Academics who tweet: "messy" identities in academia. Journal of Applied Research in Higher Education, 8(2), 210221. doi: 10.1108/JARHE-11-2014-0114
Bucchi, M. (2008). Of deficits, deviations and dialogues: Theories of public communication of science. En: M. Bucchi \& B. Trench (Eds.), Handbook of public communication of science and technology (pp. 57-76). London: Routledge.

Evans, A. (2016). Stance and identity in Twitter hashtags. Language@Internet, 13(1).urn:nbn:de:0009-7-43402

Fairclough, N. (2006). Discourse and Social Change. Cambridge: Polity Press.

García Negroni, M. M. (2008). Subjetividad y discurso científico-académico: Acerca de algunas manifestaciones de la subjetividad en el artículo de investigación en español. Revista Signos, 41(66), 9-31. doi:https://dx.doi.org/10.4067/S071809342008000100001

Georgakopoulou, A. (2015). Small Stories Research: Methods-Analysis-Outreach. En: A. De Fina \& A. Georgakopoulou. The Handbook of Narrative Analysis (pp. 255-271). Chichester: John Wiley \& Sons.

Gimenes, F. \& Mira, C. (2019). O ethos discursivo em interações de um grupo de apoio a familiares de pessoas com a Doença de Alzheimer. Revista DisSoL - Discurso, Sociedade e Linguagem, 5(9), 195-212 . Recuperado de: http://ojs.univas.edu. br/index.php/revistadissol/article/view/606

Ghosh, P., Schwartz, G. \& Narouze, S. (2020). Twitter as a powerful tool for communication between pain physicians during COVID-19 pandemic. Regional Anesthesia \& Pain Medicine, 46(2), 1. doi: 10.1136/ rapm-2020-101530

Gonsalves, C.A., McGannon, K. R., \& Pegoraro,A. (2019). Acritical discourse analysis of gendered cardiovascular disease meanings of the \#MoreMoments campaign on Twitter. Journal of Health Psychology. Publicación anticipada en línea. doi:10.1177/1359105319878240

López-Goñi, I., \& Sánchez-Angulo, M. (2018). Social networks as a tool for science communication and public engagement: focus on Twitter, FEMS Microbiology Letters, 365(2), 1-4. doi:https://doi. org/10.1093/femsle/fnx246

López-Pujalte, C., \& Nuño-Moral, M.V. (2020). La "infodemia" en la crisis del coronavirus: Análisis de desinformaciones en España y Latinoamérica. Revista Española de Documentación Científica, 43(3), 1-22. doi:10.3989/redc.2020.3.1807 
Maingueneau, D. (2002). Problèmes d'ethos. Pratiques: linguistique, littérature, didactique, 113-114, 5567. doi:10.3406/prati.2002.1945

Morandeira Rivas, A., Riquelme Gaona, J., Álvarez Gallego, M., Targarona Soler, E.M., \& Moreno Sanz, C. (2018). Uso de las redes sociales por parte de los cirujanos generales. Resultados de la encuesta nacional de la Asociación Española de Cirujanos. Cirugía Española, 97(1), 1-19. doi: 10.1016/j. ciresp.2018.07.001

Patel, S. (2019). Science and social media: Opportunities, benefits and risks. Science Communication in South Africa: Reflections on Current Issues (pp. 109-149). Cape Town: African Minds.

Pérez-Dasilva, J.A., Meso-Ayerdi, K., \& MendigurenGaldospín, T. (2020). Fake news y coronavirus: detección de los principales actores y tendencias a través del análisis de las conversaciones en Twitter. El Profesional de la Información, 29(3), e290308. doi:https://doi.org/10.3145/epi.2020.may.08

Pérez-Rodríguez, A., González-Pedraz, C., \& Alonso Berrocal, J. (2018). Twitter como herramienta de comunicación científica en España. Principales agentes y redes de comunicación. Communication Papers, 7(13), 95-112. doi:http://dx.doi.org/10.33115/ udg_bib/cp.v7i13.21986

Pollett, S., \& Rivers, C. (2020). Social Media and the New World of Scientific Communication during the COVID-19 Pandemic, Clinical Infectious Diseases, ciaa553, doi: 10.1093/cid/ciaa553

Ramírez, C. (2013). Ethos and Critical Discourse Analysis: From Power to Solidarity. COnTEXTES Revue de sociologie de la littérature, 13, 1-17. doi:10.4000/ contextes.5805

Rochwerg, B., Parke, R., Murthy, S., Fernando, S., Leigh, J.P., Marshall, J., ... Sevransky, J. (2020). Misinformation during the Coronavirus Disease 2019 Outbreak: How Knowledge Emerges From Noise. Critical Care Explorations, 2(4), 1-6. doi:10.1097/ CCE.0000000000000098

Rosenberg, H., Syed, S., \& Rezaie, S. (2020). The Twitter pandemic: The critical role of Twitter in the dissemination of medical information and misinformation during the COVID-19 pandemic. CJEM, 22(4), 418-421. doi:10.1017/cem.2020.361
Salvador Mata, B., Raffio, V., \& Cortiñas-Rovira, S. (2020). Análisis cualitativo de la percepción de las pseudociencias en el colectivo médico español. Revista Española de Comunicación en Salud, 11(1), 71-78. doi:http://dx.doi.org/10.20318/ recs.2020.5051

Wahbeh, A., Nasralah, T., Al-Ramahi, M., \& El-Gayar, O. (2020). Mining Physicians' Opinions on Social Media to Obtain Insights Into COVID-19: Mixed Methods Analysis. JMIR Public Health Surveillance 6(2), 1-10. doi:10.2196/19276

Wicke, P. \& Bolognesi, MM. (2020). Framing COVID-19: How we conceptualize and discuss the pandemic on Twitter. PLoS ONE, 15(9), 1-24. doi: 10.1371/journal. pone. 0240010 
88 // Manco Vega

\section{Anexo}

A. Listado de tweets analizados.

\begin{tabular}{|c|c|c|}
\hline Cuenta & $\begin{array}{l}\text { Número de tweets } \\
\text { seleccionados }\end{array}$ & Tweets analizados \\
\hline @Alvaro_Taype & 16 & $\begin{array}{l}\text { https://twitter.com/Alvaro_Taype/status/1250203161899474951 } \\
\text { https://twitter.com/Alvaro_Taype/status/1258829264549527555 } \\
\text { https://twitter.com/Alvaro_Taype/status/1263298399421464578 } \\
\text { https://twitter.com/Alvaro_Taype/status/1252075742806126593 } \\
\text { https://twitter.com/Alvaro_Taype/status/1262147040613711873 } \\
\text { https://twitter.com/Alvaro_Taype/status/1262894669886902274 } \\
\text { https://twitter.com/Alvaro_Taype/status/1268294488029626370 } \\
\text { https://twitter.com/Alvaro_Taype/status/1268765137617518592 } \\
\text { https://twitter.com/Alvaro_Taype/status/1253227840973279233 } \\
\text { https://twitter.com/Alvaro_Taype/status/1249061164044165120 } \\
\text { https://twitter.com/Alvaro_Taype/status/1245143250970738688 } \\
\text { https://twitter.com/Alvaro_Taype/status/1272581844404514819 } \\
\text { https://twitter.com/Alvaro_Taype/status/1260620130536566786 } \\
\text { https://twitter.com/Alvaro_Taype/status/1260637876456435712 } \\
\text { https://twitter.com/Alvaro_Taype/status/1269371270031986689 } \\
\text { https://twitter.com/Alvaro_Taype/status/1273068661956317184 }\end{array}$ \\
\hline @drhuerta & 7 & $\begin{array}{l}\text { https://twitter.com/drhuerta/status/1264347595670990853 } \\
\text { https://twitter.com/drhuerta/status/1264998824742989824 } \\
\text { https://twitter.com/drhuerta/status/1263679059365711875 } \\
\text { https://twitter.com/drhuerta/status/1253825155324956673 } \\
\text { https://twitter.com/drhuerta/status/1261288716422742016 } \\
\text { https://twitter.com/drhuerta/status/1264761635346034690 } \\
\text { https://twitter.com/drhuerta/status/1268968195043536898 }\end{array}$ \\
\hline @emezones & 4 & $\begin{array}{l}\text { https://twitter.com/emezones/status/1265003317769515009 } \\
\text { https://twitter.com/emezones/status/1244109742236209159 } \\
\text { https://twitter.com/emezones/status/1242596191017160704 } \\
\text { https://twitter.com/emezones/status/1264749146113585153 }\end{array}$ \\
\hline @ErnesBustamante & 4 & $\begin{array}{l}\text { https://twitter.com/ErnesBustamante/status/1258760409739386880 } \\
\text { https://twitter.com/ErnesBustamante/status/1257664768887992321 } \\
\text { https://twitter.com/ErnesBustamante/status/1268737015690002432 } \\
\text { https://twitter.com/ErnesBustamante/status/1257681840007192578 }\end{array}$ \\
\hline @jjaimemiranda & 2 & $\begin{array}{l}\text { https://twitter.com/jjaimemiranda/status/1261250346724147202 } \\
\text { https://twitter.com/jjaimemiranda/status/1257160765896749057 }\end{array}$ \\
\hline
\end{tabular}

